\title{
A Note on Fuzzy Volterra-Fredholm Integro-Integral Equations
}

\author{
Fan Guanghui* \\ Department of Mathematics, Heilongjiang Institute of Technology, Harbin, China, 150050. \\ * Corresponding author. Email: fghxyz@163.com \\ Manuscript submitted July 10, 2016; accepted September 30, 2016. \\ doi: 10.17706/ijapm.2017.7.1.59-68
}

\begin{abstract}
In this paper we studied a class of Volterra integral equation by using Mönch fixed point theorem, we intend to offer new numerical methods to solve the fuzzy Volterra- Fredholm integro-integral equations. By using the comparison theorems and Picard iterated approximation method we obtained the existence theorem of the solution under some weaker conditions, and we proved the theorem. Some examples are investigated to verify convergence results and to illustrate the efficiently of the method.
\end{abstract}

Key words: Fuzzy integral equations, fuzzy number space, measure of noncompactness, mönch fixed point theorem.

\section{Introduction}

The definition and properties of fuzzy differential and integral have been discussed by many people see [1]-[4]. Dubois and Prade [5]-[7] are the earlier and more all-around among them. Kaleva [8]-[10] discussed the properties of fuzzy different and integral, meanwhile he studied Cauchy problem of fuzzy differential equation. Seikala [11], [12] discussed the solvability of fuzzy initial value problem basing on the special structure of E1. Park et. al. [13]-[17] and Song [18]-[21] studied existence and uniqueness theorem of the solution of other type of fuzzy differential equations [FDE] and fuzzy integral equations [FIE].

Due to different understand to fuzzy number space (the complete metric space; the cone of some Banach space; The family of the interval sets) and different methods to deal with equations, There are different methods to discuss FDE and FIE. The methods of [16], [17], [21] is basing on Picaerd successive iteration or comparison theorems, [20] used embedding theorem of $\mathrm{E}^{\mathrm{n}}$, so it can use some subtle method such as maximum and minimum method. Seikala [22] utilized special structure of $\mathrm{E}^{1}$, deal with FDE (I) by transferring FDE to infinite system of ODE.

In [16], [17], [20] Volterra integral equation were also discussed. In [16], [17] it is studied by using comparison theorems and Picard iterated approximation method, in [20] it is studied by Darbo fixed point theorem. Moreover [16] researched indeed to the fuzzy functional integral equation. In this paper we studied a class of Volterra integral equation by using Mönch fixed point theorem, and obtained the existence theorem of the solution.

In this paper the conclusions above is extended under the weaker conditions.

\section{Procedure for Paper Submission Preliminarues}

$\mathrm{P}_{k}\left(\mathrm{R}^{\mathrm{n}}\right)-\left\{\mathrm{A} \subset \mathrm{R}^{\mathrm{n}}\right\} \mathrm{A}$ is nonempty compact convex subsets of $\mathrm{R}^{\mathrm{n}},{ }_{b(f) c}\left(\mathrm{R}^{\mathrm{n}}\right)-\left\{\mathrm{A} \subset \mathrm{R}^{\mathrm{n}}\right\} \mathrm{A}$ is nonempty 
bounded(closed)convex subsets of $\mathrm{R}^{\mathrm{n}}$.

and define the addition and scalar multiplication in $\mathrm{P}_{\mathrm{k}}\left(\mathrm{R}^{\mathrm{n}}\right)$ as usual. Let $I=[0, a] \subset \mathrm{R},(a>0)$ be a compact interval and denote $E^{n}=\left\{u: \mathrm{R}^{n} \rightarrow[0,1]\right.$ |where $u$ satisfies (i) - (iv) below $\}$,

1) $u$ is normal, i.e. there exists an $x_{0} \in \mathrm{R}^{n}$, such that $u\left(x_{0}\right)=1$;

2) $u$ is fuzzy convex, i.e. $u(\lambda x+(1-\lambda) y) \geq \min \{u(x), u(y)\}, x, y \in \mathrm{E}^{n}, \lambda \in[0,1]$;

3) $u$ is upper semicontinuous;

4) $[u]^{0}=\operatorname{cl}\left\{x \in \mathrm{R}^{n} \mid u(x)>0\right\}$ is compact.

If $u \in E^{n}$, then $u$ is called a fuzzy number and $E^{n}$ is said to be a space of fuzzy numbers.

For $0<\alpha \leq 1$, denote $[u]^{\alpha}=\left\{x \in \mathrm{R}^{n} \mid u(x) \geq \alpha\right\}$. Then from (i)-(iv), it follows that the $\alpha$-level set $[u]^{\alpha} \in \mathrm{P}_{k}\left(\mathrm{R}^{n}\right)$, for all $0 \leq \alpha \leq 1$.

For the addition and scalar multiplication in $E^{n}$, we have

$$
[u+v]^{\alpha}=[u]^{\alpha}+[v]^{\alpha}, \quad[k u]^{\alpha}=[k v]^{\alpha},
$$

where $u, v \in E^{n}, k \in \mathrm{R}, 0 \leq \alpha \leq 1$. Define $D: E^{n} \times E^{n} \rightarrow[0, \infty)$ by the equation :

$$
D(u, v)=\sup _{0 \leq \alpha \leq 1} d\left([u]^{\alpha},[v]^{\alpha}\right)
$$

where $d$ is the Hausdorff metric defined in $\mathrm{P}_{k}\left(\mathrm{R}^{n}\right)$. Then it is easy to see that $D$ is a metric in $E^{n}$.

Further, using the results in [16], we know that

1) $\left(E^{n}, D\right)$ is a complete metric space;

2) $\left(E^{n}, D\right)$ is invariant, i.e. $D(u+w, v+w)=D(u, v)$ for all $u, w \in E^{n}$;

3) $D(k u, k v)=|k| D(u, v)$ for all $u, v \in E^{n}, k \in \mathrm{R}$.

Now, we recall some integrability properties in [10] for the fuzzy set-valued mappings of a real variable whose values are in $\left(E^{n}, D\right)$.

Definition 2.1. We say that a mapping $F: I \rightarrow E^{n}$ is strongly measurable, if for all $\alpha \in[0,1]$ the set-valued mapping $F^{\alpha}: I \rightarrow \mathrm{P}_{k}\left(\mathrm{R}^{n}\right)$ defined by

$$
F^{\alpha}(t)=[F(t)]^{\alpha}
$$

is (Lebesgue) measurable, when $\mathrm{P}_{k}\left(\mathrm{R}^{n}\right)$ is endowed with the topology generated by the Hausdorff metric

A mapping $F: I \rightarrow E^{n}$ is called integrably bounded if there exists an integrable function $h$ such that $\|x\| \leq h(t)$,for all $x \in F_{0}(t)$.

Definition 2.2. Let $F: I \rightarrow E^{n}$. The integral of $F$ over $I$, denoted by $\int_{I} F(t) d t$, is defined -levelwise by the equation

$$
\left[\int_{I} F(t) d t\right]^{\alpha}=\int_{I} F_{\alpha}(t) d t=\left\{(\mathrm{L}) \int_{I} f(t) d t \mid f: \mathrm{I} \rightarrow \mathrm{R}^{\mathrm{n}} \text { is a measurable selection for } F^{\alpha}\right\} \text {,for all } 0<\alpha \leq
$$

1.

A strongly measurable and integrably bounded mapping $F: I \rightarrow E^{n}$ is said to be integrable over $I$, if 
$\int_{I} F(t) d t \in E^{n}$

Proposition 2.1. If $F: I \rightarrow E^{n}$ is strongly measurable and integrably bounded, then $F$ is integrable .

Corollary 2.1. If $F: I \rightarrow E^{n}$ is continuous, then it is integrable .

Proposition2.2. Let $F, G: I \rightarrow E^{n}$ be integrable and $c \in I$. Then

$$
\int_{0}^{a} F(t) d t=\int_{0}^{c} F(t) d t+\int_{c}^{a} F(t) d t
$$

Proposition 2.3. Let $F, G: I \rightarrow E^{n}$ be integrable and $\lambda \in \mathrm{R}$. Then

1) $\int_{I}(F(t)+G(t)) d t=\int_{I} F(t) d t+\int_{I} G(t) d t$;

2) $\int_{I} \lambda F(t) d t=\lambda \int_{I} F(t) d t$

3) $D(F, G)$ is integrable;

4) $\quad D\left(\int_{I} F(t) d t, \int_{I} G(t) d t\right) \leqslant(\mathrm{L}) \int_{I} D(F(t), G(t)) d t$.

Furthermore, we list the embedding theorem in [11] on the space $\left(E^{n}, D\right)$.

C. There exists a real Banach space $X$ such that $E^{n}$ can be embedding as a convex cone $C$ with vertex $O$ into $X$. Furthermore, the following conditions hold true:

1) the embedding $j$ is isometric;

2) addition in $X$ induces addition in $E^{n}$;

3) multiplication by nonnegative real number in $X$ induces the corresponding operation in $E^{n}$;

4) $C-C$ is dense in $X$;

5) $C$ is closed.

Remark 2.1. In this paper, we always denote $X$ to be the Banach space in Proposition $2.4, j$ the isometric embedding from $\left(E^{n}, D\right)$ into $X$.

Let $\left(E^{n}, D\right)$ be the space of fuzzy numbers, $E_{3}^{n}=E^{n} \times E^{n} \times E^{n}, \quad[I=0, a], \quad a \geq 0 \quad$ and $\mathrm{D}=\left\{(t, s) \in \mathrm{R}^{2} \mid 0 \leq s \leq t \leq a\right\}, \mathrm{D}_{0}=I \times I$. The fuzzy number $\hat{\theta}$ is defined by

$$
\hat{\theta}(r)= \begin{cases}1 & r=0 . \\ 0 & r \neq 0,\end{cases}
$$

and define function $\theta=\theta(t) \equiv \hat{\theta}$, for all $t \in \mathrm{I}$.

Let $C\left(I, E^{n}\right)$ denote the space of continuous fuzzy set-valued mappings from $I$ into $E^{n}$. The addition and nonnegative scalar multiplication in $C\left(I, E^{n}\right)$ are induced, respectively, by the corresponding operations in $\mathrm{E}^{n}$. Then it is easy to see that $C\left(I, E^{n}\right)$ is a convex cone.

We know that the space $C\left(I, E^{n}\right)$ with the metric

$$
D(u, \theta)=\sup _{t \in I} D(u(\mathrm{t}), \hat{\theta}), u \in \mathrm{C}\left(I, E^{n}\right),
$$

becomes complete metric space. It has analogous results for the space $C\left(I \times E^{3}, E^{n}\right)$. 
By Proposition 2.4, we know that the embedding $j$ from $\left(E^{n}, D\right)$ onto its range $j\left(E^{n}\right) \subset X$ is isometrically isomorphism, and so the embedding

$$
j: \quad \mathrm{C}\left(I, E^{n}\right) \rightarrow \mathrm{C}\left(I, j\left(E^{n}\right)\right) \subset \mathrm{C}(I, X)
$$

is also isometrically isomorphism.

Let $\mathrm{C}(I, X)$ denote the Banach space of abstract continuous function from $I$ to $X$, and the norm

$$
\|x\|=\max _{\mathrm{t} \in \mathrm{I}}\|x(t)\| .
$$

For $H \subset \mathrm{C}(I, X)$, we denote

$$
\begin{gathered}
H(t)=\{x(t) \mid x \in H\} \subset X, \\
H(I)=\{x(t) \mid x \in H, t \in I\}=\bigcup_{t \in I} H(t) \subset X .
\end{gathered}
$$

Definition 2.3. Let $A$ be a bounded subset of $X$, the Kuratowski measure of noncompactness is defined by

$\alpha(A)=\inf \{\varepsilon>0: A$ can covered by a finite number of sets each with diameter $\leq \varepsilon\}$.

Proposition 2.6. Let $\alpha$ be the Kuratowski measure of noncompactness and suppose that $A$ and $B$ are two arbitrary bounded subsets of $X$, then we have

1) $\alpha(A)=0$ iff. $A$ is relatively compact;

2) $\alpha(A) \leq \alpha(B), \quad$ if $A \subseteq B$;

3) $\alpha(A)=\alpha(\overline{\operatorname{co}}(A))$, where $\operatorname{co}(A)$ denotes the convex hull of $A$;

4) $\alpha(A \cup B)=\max \{\alpha(A), \alpha(B)\}$;

5) $\alpha(t A)=|t| \quad \alpha(A)$, where $t A=\{t x: x \in A\}$;

6) $\alpha(A+B) \leq \alpha(A)+\alpha(B)$, where $A+B=\{x+y \mid x \in A$ and $y \in B\}$.

Proposition 2.7. Suppose $H \subset \mathrm{C}(I, X)$ be bounded and equicontinuous, then

1) $\alpha(H)=\alpha(H(\mathrm{I}))$;

2) $\alpha(H(I))=\max _{\mathrm{t} \in \mathrm{I}} \alpha(H(t))$.

Corollary 2.2. Suppose $A \subset X$ be bounded, the mapping $f: I \times A \rightarrow X$ is bounded and uniform continuous. Then

$$
\alpha(f(I \times B))=\max _{t \in \mathrm{I}} \alpha(f(t, B)), \quad \forall B \subset A .
$$

In this section, we suppose that $(X,\|\cdot\|)$ is a real Banach space .

Let $I=[0, a], a>0, \mathrm{D}=\left\{(t, s) \in R^{2} \mid 0 \leq s \leq t \leq a\right\}$ and $D_{0}=I \times I$.

The space of continuous functions $\mathrm{C}(I, X)=\{x \mid x: I \rightarrow X$ is continuous $\}$ with a norm $\|x\|_{C}=\max _{t \in I}\|x(t)\|$ becomes Banach space.

Lemma 2.1 [13]. Let $B_{1}, B_{2} \subset \mathrm{C}(I, X)$ be two countable sets and for every $x_{0} \in \mathrm{C}(I, X)$ and $\overline{B_{1}}=\overline{c o}\left(\left\{x_{0}\right\} \cup B_{2}\right)$, then $\overline{B_{1}(t)}=\overline{c o}\left(\left\{x_{0}(t)\right\} \cup B_{2}(t)\right)$, for all $t \in I$.

Lemma 2.2 [9]. Let $\mathrm{B}$ be the countable set of strongly measurable functions $x: I \rightarrow X$. If there is 
$m \in \mathrm{L}\left(I, R^{+}\right) \quad$ such that

$$
\|x(t)\| \leq m(t), \quad \text { a.e. } \quad t \in I, \quad x \in B,
$$

then $\alpha(B(t)) \in \mathrm{L}\left(I, R^{+}\right)$and $\alpha\left(\left\{\int_{I} x(t) d t \mid x \in B\right\}\right) \leq 2 \int_{I} \alpha(B(t)) d t$.

Lemma 2.3 [8]. Let $B \subset \mathrm{C}(I, X)$ be a equicontinuous and bounded set. Define $m(t)=\alpha(B(t))$,

$t \in I$, then $m(t)$ is continuous on $I$ and $\alpha\left(\int_{I} B(t) d t\right) \leq \int_{I} \alpha(B(t)) d t$.

Lemma 2.4[13]. Let $\mathrm{p} \in \mathrm{C}\left(\mathrm{I}, \mathrm{R}^{+}\right)$satisfies

$$
p(t) \leq M \int_{0}^{t} p(s) d s+N \int_{0}^{T} p(s) d s, \quad t \in I,
$$

where $\mathrm{M}>0, \mathrm{~N} \geq 0$ such that $\mathrm{N}\left(\mathrm{e}^{\mathrm{MT}}-1\right)<\mathrm{M}$, then $\mathrm{p}(\mathrm{t}) \equiv 0, \quad \mathrm{t} \in \mathrm{I}$.

Lemma 2.5 [4]. Let $B \subset \mathrm{C}(I, X)$ be the equicontinuous and bounded set, then $\alpha(B)=\max _{t \in I} \alpha(B(t))$, where $B(t)=\{x(t) \mid x \in B\} \subseteq X$.

Lemma 2.6. Let $\mathrm{j}$ is the embedding operator defined as Proposition 2.4, $\mathrm{c}$ is a real number and $B \subset E^{n}$, then we have

$$
\alpha(j(c B))=|c| \alpha(j B)
$$

Lemma 2.7. (Mönch Fixed Point Theorem). Let $X$ be the Banach space, $K \in \mathrm{P}_{f c}(X)$. If the pmapping $F: K \rightarrow K$ satisfies the following conditions

$\left(C_{1}\right) F: K \rightarrow K$ is continuous

$\left(C_{2}\right) C \subset K$ is countable, $x \in X$ and $\bar{C}=\overline{c o}(\{x\} \bigcup F(C))$ implies that $\bar{C}$ is compact (i,e,C is relatively compact) , then $F$ has at least one fixed point in $K$.

\section{Math Main Results}

In this section, we shall study the following fuzzy integral equation (FIE)

$$
(\mathrm{VTS})\left\{\begin{array}{l}
u(t)=g(t)+\int_{0}^{t} k(t, s) f(s, u(s),(T u)(s),(\mathrm{Su})(s)) d s \\
T u(t)=\int_{0}^{t} p(t, s) u(s) d s \\
S u(t)=\int_{0}^{a} q(t, s) u(s) d s
\end{array}\right.
$$

where $t \in I, T: C\left(I, E^{n}\right) \rightarrow C\left(I, E^{n}\right)$ is Volterra integral operator and $S: C\left(I, E^{n}\right) \rightarrow C\left(I, E^{n}\right)$ is Fredholm integral operator .

For $B \subset C\left(I, E^{n}\right)$ and $t \in I$, let $B(t)=\{u(t) \mid u \in B\}$,

$(\mathrm{TB})(t)=\{T u(t) \mid u \in B\},(\mathrm{SB})(t)=\{S u(t) \mid u \in B\}$.

We suppose that the following conditions are satisfied for the equation (VTS).

(G) $g \in C\left(I, E^{n}\right)$, 
(KPQ) $k, p \in \mathrm{C}\left(\triangle, R^{1}\right)$ and $q \in \mathrm{C}\left(I_{0}, R^{1}\right)$,

where $\mathrm{D}=\{(t, s) \mid 0 \leq s \leq t \leq a\}$ and $\mathrm{D} 0=\mathrm{I} \times \mathrm{I}$,

By the compactness of the set $\mathrm{D}$ and D0, we have that $k_{0}=\max |k(D)|, \quad p_{0}=\max |p(D)|$, and $q_{0}=\max \left|q\left(D_{0}\right)\right|$ exist.

( $\left.\mathrm{F}_{1}\right) \quad f \in \mathrm{C}\left(I \times E_{3}^{n}, E^{n}\right)$,

( $\left.\mathrm{F}_{2}\right) \varlimsup_{R \rightarrow \infty} \frac{M(R)}{R}<\frac{1}{a k_{0}}$,

where $M(R)=\sup \left\{D(f(t, u(t),(T u)(t),(S u)(t)), \hat{\theta}) \mid D_{C}(u, \theta) \leq R\right\}$,

$\left(\mathrm{F}_{3}\right)$ For any countable equicontinuous bounded set $B \subset C\left(I, E^{n}\right)$ and $t \in I$,

$$
\alpha(j f(s, B(s),(T B)(t),(S B)(s))) \leq L_{1} \alpha(j B(s))+L_{2} \alpha(j(T B)(s))+L_{3} \alpha(j(S B)(s))
$$

where $\mathrm{j}$ is the embedding operator defined by Proposition 2.4 and $L_{i} \geq 0,(i=1,2,3)$ are the constants that satisfies one of the following conditions

$$
\begin{aligned}
& \left(L_{1}\right) a q_{0} L_{3}\left(\exp \left(2 a k_{0}\left(L_{1}+a p_{0} L_{2}\right)\right)-1\right)<L_{1}+a p_{0} L_{2} \\
& \left(L_{2}\right) a k_{0}\left(2 L_{1}+a p_{0} L_{2}+a q_{0} L_{3}\right)<1
\end{aligned}
$$

Theorem 3.1. Assume that FIE (VTS) satisfies all above conditions, then (VTS) has at least one solution in C (I, $\left.E^{n}\right)$.

Proof. Define the operators

$$
\begin{aligned}
& F[T, S] u(t)=f(t, u(t),(T u)(t),(S u)(t)), \quad t \in I, \\
& K(T, S, f, u)(t)=\int_{0}^{t} k(t, s) F[T, S] u(s) d s, \quad t \in I, \\
& A u(t)=g(t)+K(T, S, f, u), \quad t \in I .
\end{aligned}
$$

Clearly, $u \in \mathrm{C}\left(\mathrm{I}, E^{n}\right)$ is the solution of (VTS) if and only if $\mathrm{u}$ is the fixed point of the operator A in C (I, $\left.E^{n}\right)$.

At first we prove that A: $\mathrm{C}\left(\mathrm{I}, E^{n}\right) \rightarrow \mathrm{C}\left(\mathrm{I}, E^{n}\right)$ is continuous. For this, for any $u_{1}, u_{2} \in \mathrm{C}\left(\mathrm{I}, E^{n}\right), t \in I$. By the definition od the operations $\mathrm{T}$ and $\mathrm{S}$, we have

$$
\begin{aligned}
& D_{C}\left(T u_{1}, T u_{2}\right) \leq p_{0} \int_{0}^{a} D\left(u_{1}(s), u_{2}(s)\right) d s \\
& D_{C}\left(S u_{1}, S u_{2}\right) \leq q_{0} \int_{0}^{a} D\left(u_{1}(s), u_{2}(s)\right) d s
\end{aligned}
$$

hence T, $\mathrm{S}: \mathrm{C}\left(I, E^{n}\right) \rightarrow \mathrm{C}\left(I, E^{n}\right)$ is continuous. Since

$$
D\left(A u_{1}(t), A u_{2}(t)\right) \leq k_{0} \int_{0}^{a} D\left(F[T, S] u_{1}(s), F[T, S] u_{2}(s)\right) d s
$$

thus by the continuity of the mapping $\mathrm{f}$, we have that $\mathrm{A}$ is continuous. 
By the assumption $\left(\mathrm{F}_{2}\right)$, there are $r \in\left(0, \frac{1}{a k_{0}}\right)$ and $R_{0}>0$ such that

$$
M(R)<r R
$$

for all $R \geq R_{0}$.

Take $R^{*} \geq R_{0}$ and put $W=\left\{u \in \mathrm{C}\left(I, E^{n}\right) \mid D_{C}(u, g) \leq R^{*}\right.$,

$$
\left.D\left(u\left(t_{1}\right), u\left(t_{2}\right)\right) \leq D\left(g\left(t_{1}\right), g\left(t_{2}\right)\right)+r R^{*}\left[\int_{t_{1}}^{t_{2}}\left|k\left(t_{1}, s\right)\right| d s+\int_{0}^{t_{2}}\left|k\left(t_{1}, s\right)-k\left(t_{2}, s\right)\right| d s\right]\right\}
$$

then $W \in P_{b c}\left(C\left(I, E^{n}\right)\right)$ is equicontinuous and for every $u \in W, t_{1}, t_{2} \in I, t_{1} \leq t_{2}$

$$
D_{C}(A u, g) \leq a k_{0} M\left(R^{*}\right)<r a k_{0} R^{*} \leq R^{*},
$$

$$
\begin{aligned}
D\left(A u\left(t_{1}\right), A u\left(t_{2}\right)\right)= & D\left(g\left(t_{1}\right), g\left(t_{2}\right)\right)+D\left(\int_{0}^{t_{1}} k\left(t_{1}, s\right) F[T, S] u(s) d s, \int_{0}^{t_{2}} k\left(t_{2}, s\right) F[T, S] u(s) d s\right) \\
\leq & D\left(g\left(t_{1}\right), g\left(t_{2}\right)\right)+D\left(\int_{0}^{t_{1}} k\left(t_{1}, s\right) F[T, S] u(s) d s, \int_{0}^{t_{2}} k\left(t_{1}, s\right) F[T, S] u(s) d s\right) \\
+ & D\left(\int_{0}^{t_{2}} k\left(t_{1}, s\right) F[T, S] u(s) d s, \int_{0}^{t_{2}} k\left(t_{2}, s\right) F[T, S] u(s) d s\right) \\
\leq & \left.D\left(g\left(t_{1}\right), g\left(t_{2}\right)\right)+\int_{t_{1}}^{t_{2}}\left|k\left(t_{1}, s\right)\right| D(F[T, S)] u(s), \hat{\theta}\right) d s \\
+ & \left.\int_{0}^{t_{2}}\left|k\left(t_{1}, s\right)-k\left(t_{2}, s\right)\right| D(F[T, S)] u(s), \hat{\theta}\right) d s \\
\leq & D\left(g\left(t_{1}\right), g\left(t_{2}\right)\right)+\left(\int_{t_{1}}^{t_{2}}\left|k\left(t_{1}, s\right)\right| d s\right) M\left(R^{*}\right) \\
& +\left(\int_{0}^{t_{2}}\left|k\left(t_{1}, s\right)-k\left(t_{2}, s\right)\right| d s\right) M\left(R^{*}\right) \\
& \leq D\left(g\left(t_{1}\right), g\left(t_{2}\right)\right)+r R^{*}\left[\int_{t_{1}}^{t_{2}}\left|k\left(t_{1}, s\right) d s+\int_{0}^{t_{2}}\right| k\left(t_{1}, s\right)-k\left(t_{2}, s\right) \mid d s\right] .
\end{aligned}
$$

Hence $A: W \rightarrow W$ is continuous. Observe the continuity of the embedding operator $\mathrm{j}$, we know that the operator $j A: j W \rightarrow j W$ is also continuous.

Let $B=\left\{u_{n}\right\} \subset W, u_{0} \in W$ such that $\bar{B}=\overline{c o}\left(\left\{u_{0}\right\} \bigcup A(B)\right)$

Next to prove that $\overline{j B} \in P_{k}\left(j \mathrm{C}\left(I, E^{n}\right)\right)=P_{k}\left(\mathrm{C}\left(I, j E^{n}\right)\right)$.

By Lemma 2.1 we have

$$
\overline{j B(t)}=\overline{c o}\left(\left\{j u_{0}(t)\right\} \bigcup j A(B)(t)\right)=\overline{c o}\left(\left\{j u_{0}(t)\right\} \bigcup A(j B)(t)\right) .
$$

Thus by Lemma 2.2 and 2.6, the properties of measure of noncompactness and (2) we have

$$
\begin{gathered}
\alpha(j B(t))=\alpha(\overline{j B(t))}=\alpha(A(j B)(t)) \\
\quad=\alpha\left(j \int_{0}^{t} k(t, s) F[T, S] u(s) d s\right)
\end{gathered}
$$




$$
\leq 2 \int_{0}^{t} k(t, s) \mid \alpha(j F[T, S] u(s)) d s
$$

Then by the condition $\left(\mathrm{F}_{3}\right)$, Lemma2.3 and observe that TB and SB are equicontinuous bounded subset of $\mathrm{C}\left(\mathrm{I}, E^{n}\right)$ and the continuity of the operator j, we obtain that

$$
\alpha(j F[T, S] u(s)) \leq L_{1} \alpha(j B(s))+L_{2} p_{0} \int_{0}^{s} \alpha(j B(\tau)) d \tau+L_{3} q_{0} \int_{0}^{a} \alpha(j B(\tau)) d \tau
$$

By (4) and (3), we get

$$
\begin{gathered}
\alpha(j B(t)) \leq 2 L_{1} k_{0} \int_{0}^{t} \alpha(j B(s)) d s+2 L_{2} p_{0} k_{0} \int_{0}^{t} \int_{0}^{s} a(j B(\tau)) d \tau d s \\
+2 L_{3} q_{0} k_{0} \int_{0}^{t} \int_{0}^{a} \alpha(j B(\tau)) d \tau d s
\end{gathered}
$$

and then we have

$$
\begin{gathered}
\alpha(j B(t)) \leq 2 k_{0} L_{1} \int_{0}^{t} \alpha(j B(s)) d s+2 L_{2} p_{0} k_{0} \int_{0}^{t} \int_{0}^{s} a(j B(\tau)) d \tau d s+2 k_{0} q_{0} L_{3} t \int_{0}^{a} \alpha(j B(s)) d \\
\leq 2 k_{0} L_{1} \int_{0}^{t} \alpha(j B(s)) d s+2 k_{0} p_{0} L_{2} \int_{0}^{t}(t-s) \alpha(j B(s)) d s+2 k_{0} q_{0} L_{3} t \int_{0}^{a} \alpha(j B(s)) d
\end{gathered}
$$

By the equicontinuity and boundedness of $B \subset C\left(I, E^{n}\right)$, we know

$$
\mathrm{l}(\mathrm{t})=\alpha(j B(t)) \in C\left(I, R^{+}\right)
$$

1) Suppose that $L_{i} \geq 0$, i=1, 2, 3 satisfies the condition ( $\left.L_{1}\right)$, then from (6) we have

$$
l(t) \leq M \int_{0}^{t} l(s) d s+N \int_{0}^{a} l(s) d s,
$$

where $M=2 L_{1} k_{0}+2 p_{0} k_{0} L_{2}, \quad N=2 q_{0} k_{0} L_{3}$.

By Lemma 2.4, it follows $\mathrm{l}(\mathrm{t}) \equiv 0, \quad \mathrm{t} \in \mathrm{I}$.

2) Let $L_{i} \geq 0, i=1,2,3$ satisfies (ii) in $\left(H_{2}\right)$, also from (6) we have

$$
\begin{gathered}
\int_{0}^{a} l(s) d s \leq \int_{0}^{a}\left[2 k_{0} L_{1}+2 p_{0} k_{0} L_{2} t+2 q_{0} k_{0} L_{1} t\right] d t \int_{0}^{a} l(s) d s \\
=\left[2 a k_{0} L_{1}+a^{2} p_{0} k_{0} L_{2}+a^{2} q_{0} k_{0} L_{3}\right] \int_{0}^{a} l(s) d s \\
=a k_{0}\left[2 L_{1}+a p_{0} L_{2}+a q_{0} L_{3}\right] \int_{0}^{a} l(s) d s .
\end{gathered}
$$

Thus $\int_{0}^{a} l(t) d t=0$, hence $\mathrm{l}(\mathrm{t}) \equiv 0, \quad \mathrm{t} \in \mathrm{I}$,

By Lemma 2.5, both (i) and (ii) follows that

$$
\alpha(j B)=\max _{t \in I} \alpha(j B(t))=0,
$$


therefore we have $\overline{j B} \in P_{k}\left(C\left(I, j E^{n}\right)\right)=j P_{k}\left(C\left(I, E^{n}\right)\right)$.

It show by Lemma 2.7 the operator $j A: j W \rightarrow j W$ has at least one fixed point $v^{*}$ in $j W \subset \mathrm{C}\left(I, j E^{n}\right)$. Put $v^{*}=j\left(u^{*}\right)$ for some $u^{*} \in W$, thus $u^{*}$ is the (global) solution of (VTS) in $\mathrm{C}\left(I, E^{n}\right)$.

\section{Acknowledgment}

This work is supported by Foundation of Department of Education of Heilongjiang Province under Grant 12541678.

\section{References}

[1] Aumann, R. J. (1965). Integrals of set-valued function. J. Math. Anal. Appl., 12, 1-12.

[2] Casting, G., \& Valadier, M. (1977). Convex Analysis and Measurable Multi-functions. Springer, Berlin.

[3] Debreu, G. (1966). Integration of correspondence. Proceedings of 5th Berkeley Symp. Math. Statist. Probability (pp. 351-372). Univ .California Press, Berkeley, CA.

[4] Deimling, K. (1985). Nonlinear Functional Analysis. Springer-Verlag, Berlin.

[5] Dubois, D., \& Prade, H. (1982). Towards fuzzy differential calculus, Part 1: Integration of fuzzy mappings. Fuzzy Sets and Systems, 8.1-17/

[6] Dubois, D., \& Prade, H. (1982). Towards fuzzy differential calculus, Part 2:Integration of fuzzy mappings. Fuzzy Sets and Systems, 8, 105-116.

[7] Dubois, D., \& Prade, H. Fuzzy Sets and Systems: Theory and Application. Academic Press.

[8] Guo, D. J. (1991). External solutions of nonlinear Fredholm integral equations in ordered Banach spaces. Northeastern Math. J., 9, 416-423.

[9] Heinz, H. P. (1983). On behavior of measures of noncompactness with respect to differentiation and integration of vector value functions. Nonlinear Anal., 12, 1351-1371.

[10] Kaleva, O. (1987). Fuzzy differential equations. Fuzzy Sets and Systems, 24, 301-317.

[11] Kaleva, O. (1990). The Cauchy problem for fuzzy differential equations. Fuzzy Sets and Systems, 35, 389-396,

[12] Lakshmikantham, V., \& Lela, S. (1981). Nonlinear Differential Equations in Abstract Spaces. Pergamon Press, New York.

[13] Liu, L. S. (1996). Iterative method for solutions and coupled equasi-solutions of nonlinear Fredholm integral equations in ordered Banach spaces. Indian J. Pure Appl. Math, 27, 959-972.

[14] Martin, R. H. (1976). Nonlinear Operators and Differential Equations in Banach Spaces. Wiley, New York.

[15] Negoita, C., \& Ralescu, V. D. A. (1975). Applications of Fuzzy Sets to Systems Analysis. Wiley, New York.

[16] Park, J. Y., Lee, S. Y., \& Jeong, J. U. (2000). The approximate solutions of fuzzy functional integral equations. Fuzzy Sets and Systems, 110, 79-90.

[17] Park, J. Y., \& Jeong, J. U. (1999). A note on fuzzy integral equations. Fuzzy Sets and Systems, 108, 193-200.

[18] Puri, M. L., \& Ralescy, D. A. (1986). Fuzzy random variables. J. Math. Anal. Appl., 114,409-422.

[19] Rădstrǒm, H. (1965). An embedding theorem for spaces of convex set. Proceedings of Amer. Math. Soc. (pp. 165-169).

[20] Song, S., Liu, Q., \& Xu, Q. (1999). Existence and comparison theorems to Volterra fuzzy integral equation in $\left(\mathrm{E}^{\mathrm{n}}, \mathrm{D}\right)$. Fuzzy Sets and Systems, 104, 315-321.

[21] Song, S., \& Wu, C. (2000). Existence and uniqueness of solutions to Cauchy problem of fuzzy differential 
equations. Fuzzy Sets and Systems, 110, 55-67.

[22] Seikkala, S. (1987). On the fuzzy initial value problem. Fuzzy Sets and Systems, 24, 319-330.

Fan Guanghui was born in April 1971. He is a PhD of HIT. His major interesting is the study of mathematics and applied mathematics. He is also interested in the optimization algorithm and system modeling in research work. 